\title{
From pair correlations to pair interactions: An exact relation in one-dimensional systems
}

\author{
H. Hansen-Goos ${ }^{1,2}$, C. Lutz ${ }^{3}$, C. Bechinger ${ }^{3}$ and R. Roth ${ }^{1,2}$ \\ 1 Max-Planck-Institut für Metallforschung-Heisenbergstr. 3, 70569 Stuttgart, Germany \\ 2 ITAP, Universität Stuttgart - Pfaffenwaldring 57, 70569 Stuttgart, Germany \\ 3 2. Physikalisches Institut, Universität Stuttgart \\ Pfaffenwaldring 57, 70569 Stuttgart, Germany
}

PACS. 05.20.Jj - Statistical mechanics of classical fluids.

PACS. 82.70.Dd - Colloids.

\begin{abstract}
We demonstrate that the pair potential $V(r)$ in one-dimensional systems can be obtained analytically from the pair-distribution function in case of short-ranged interactions. We re-examine the exact statistical mechanics solution and propose a simple procedure for extracting the pair potential and the equation of state from experimentally measured pairdistribution functions. The method is applied to charge-stabilised colloids which are confined to a one-dimensional laser trap.
\end{abstract}

Any description of macroscopic properties of solids and liquids typically requires information about the microscopic interactions of the system. Particularly in dense systems, however, it is a challenging task to access these interactions due to many-body contributions. During the last decades, several approaches have been developed aiming at extracting $V(r)$ in many particle systems. Most methods utilise the pair-distribution function $g(r)$ or the structure factor $S(q)$ which are experimentally accessible either by analysing real space data or by scattering experiments. Common to all theoretical approaches in $d=2$ or $d=3$ such as density functional theory [1], Ornstein-Zernike formalism (OZ) [2], and inverse Monte Carlo (IMC) simulations [3], however, is the fact that they require more or less far-reaching assumptions which can have uncontrolled effects on the derived pair-interaction potentials. The solution of the OZ integral equations requires additional closure relations whose accuracy depends on the range of $V(r)$. In case of IMC, an arbitrarily chosen cut-off in the pair potential has to be introduced. It has been demonstrated that this can lead to artificial features (e.g., a minimum in $V(r)$ for entirely repulsive systems) [4].

In $d=1$ the situation is more favourable. In the early 1950s Gürsey [5] and Salsburg et al. [6] obtained an analytic solution for the equation of state and the pair-distribution function for systems of identical particles with arbitrary next-neighbour interaction. This exact solution can be employed, as we show in the following, to extract the pair potential $V(r)$ unambiguously from measured pair-distribution functions $g(r)$, if the interaction is sufficiently short ranged. To this end, we recall some exact results, adopting the notation of Salsburg et al., and show how the exact pair-distribution function can be calculated efficiently. 
The pressure $p$ in the system at a fixed bulk particle number density $\rho_{\mathrm{b}}$ is given by

$$
\rho_{\mathrm{b}}=-\frac{\Omega(\beta p)}{\Omega^{\prime}(\beta p)},
$$

where $\beta=1 /\left(k_{\mathrm{B}} T\right)$ is the inverse temperature, $k_{\mathrm{B}}$ Boltzmann's constant, and

$$
\Omega(s)=\int_{0}^{\infty} \exp [-s r-\beta V(r)] \mathrm{d} r,
$$

with the pair potential $V(r) . \Omega^{\prime}$ in eq. (1) denotes the derivative of $\Omega$ with respect to its argument. In an isotropic system, $g(r)$ is defined through

$$
g\left(r=\left|R-R^{\prime}\right|\right)=\frac{1}{\rho_{\mathrm{b}}^{2}}\left\langle\sum_{k=1}^{N} \sum_{(j \neq k)=1}^{N} \delta\left(R_{k}-R\right) \delta\left(R_{j}-R^{\prime}\right)\right\rangle .
$$

Here $\langle\cdot\rangle$ stands for the thermal average and $R_{k}, R_{j}$ denote the position of the $k$-th and the $j$-th particle, respectively. A quantity closely related to $g(r)$ is the one-body density profile $\rho^{(1)}(r)=\rho_{\mathrm{b}} g(r)$ of the fluid in the external field exerted by one particle fixed at the origin, $r=0$. In the following, we shall concentrate on the latter quantity, which is given by

$$
\rho^{(1)}(r)=\sum_{k=1}^{\infty} \frac{e^{-\beta p r}}{[\Omega(\beta p)]^{k}} \frac{1}{2 \pi i} \int_{\beta p-i \infty}^{\beta p+i \infty} e^{r s}[\Omega(s)]^{k} \mathrm{~d} s .
$$

We consider a short-ranged pair potential with a hard-core contribution of the form

$$
V_{\mathrm{sr}}(r)= \begin{cases}\infty & \text { if } r<\sigma \\ V(r) & \text { if } \sigma \leq r \leq \lambda \\ 0 & \text { if } r>\lambda\end{cases}
$$

where $\sigma$ is the diameter of the particle. One can show that for $V_{\mathrm{sr}}(r)$ the integrals in eq. (4) vanish if $r<k \sigma$. This allows us to introduce the Heaviside function $\Theta$ which leads to

$$
\rho^{(1)}(r)=\sum_{k=1}^{\infty} \Theta(r-k \sigma) \frac{1}{2 \pi i} \int_{\beta p-i \infty}^{\beta p+i \infty} e^{r(s-\beta p)}[\Omega(s) / \Omega(\beta p)]^{k} \mathrm{~d} s,
$$

where we have rearranged the factors. For a convenient numerical calculation of $\rho^{(1)}(r)$, we substitute $s-\beta p=i t$ which shifts the integration path onto the real axis. The resulting integral is of the Fourier type. $\Omega(\beta p+i t)$, which appears in the integrand, can be rewritten as a Fourier integral according to eq. (2) and we obtain a simple form for $\rho^{(1)}(r)$ :

$$
\rho^{(1)}(r)=\sum_{k=1}^{\infty} \Theta(r-k \sigma) \mathcal{F}_{\mathrm{p}}^{-1}\left\{\mathcal{F}_{\mathrm{p}}\left\{\exp \left[-\beta p r^{\prime}-\beta V_{\mathrm{sr}}\left(r^{\prime}\right)\right] / \Omega(\beta p)\right\}^{k}\right\} .
$$

$\mathcal{F}_{\mathrm{p}}$ denotes a restricted Fourier transformation and $\mathcal{F}_{\mathrm{p}}^{-1}$ its inverse, which are given by

$$
\mathcal{F}_{\mathrm{p}}\left\{f\left(r^{\prime}\right)\right\}=\int_{0}^{\infty} e^{-i t r^{\prime}} f\left(r^{\prime}\right) \mathrm{d} r^{\prime} \quad \text { and } \quad \mathcal{F}_{\mathrm{p}}^{-1}\{q(t)\}=\frac{1}{2 \pi} \int_{-\infty}^{+\infty} e^{i t r} q(t) \mathrm{d} t .
$$

In the numerical procedure we solve, in a first step, eq. (1) for the pressure $\beta p$. Then we calculate the function $q(t)=\mathcal{F}_{\mathrm{p}}\left\{\exp \left[-\beta p r^{\prime}-\beta V_{\mathrm{sr}}\left(r^{\prime}\right)\right] / \Omega(\beta p)\right\}$ using the fast Fourier 
transformation. We know from eq. (7) that, for the knowledge of $\rho^{(1)}(r)$ for $r \leq r_{\max }$, only the first $n_{\max }=\left\lfloor r_{\max } / \sigma\right\rfloor$ integrals are required. The sum of the integrands is a geometric sequence and one obtains

$$
\rho^{(1)}(r)=\mathcal{F}_{\mathrm{p}}^{-1}\left\{\frac{q(t)\left(1-q(t)^{n_{\max }}\right)}{1-q(t)}\right\} .
$$

Since $|q(t)|<1$ for $t \neq 0$, it is tempting to take the limit $n_{\max } \rightarrow \infty$ in eq. (9). Indeed, $q(t) /(1-q(t))$ diverges like $\rho_{\mathrm{b}} /(i t)$ at $t=0$ as required to assure that $\rho^{(1)}(r \rightarrow \infty)=\rho_{\mathrm{b}}$ is obtained. Therefore, the divergence-free expression of eq. (9) for general $r$ reads $\rho^{(1)}(r)=$ $\rho_{\mathrm{b}}+\mathcal{F}_{\mathrm{p}}^{-1}\left\{q(t) /(1-q(t))-\rho_{\mathrm{b}} /(i t)\right\}$. It turns out, however, that this expression is numerically less stable than eq. (9) which avoids the divergence at $t=0$ by truncating the sum in eq. (4).

While the calculation of the exact $\rho^{(1)}(r)$ can be performed easily for arbitrary distance $r$ numerically, it is most instructive to take a closer look at the form of $\rho^{(1)}(r)$ in the interval $[0,2 \sigma]$ (cf. ref. [7]), which can be inferred directly from eq. (7) to be

$$
\begin{array}{ll}
\rho^{(1)}(r)=0 & \text { for } 0 \leq r<\sigma \\
\rho^{(1)}(r)=\exp [-\beta p r-\beta V(r)] / \Omega(\beta p) & \text { for } \sigma \leq r \leq 2 \sigma .
\end{array}
$$

The first equation expresses the core condition. The second identity is very simple due to the fact that in the interval $\sigma \leq r \leq 2 \sigma$ one can find only the next neighbour. This particle feels on the one side the external field $V(r)$ exerted by the fixed particle at $r=0$ and on the other side the rest of the system that pushes it toward the origin with the pressure $p$.

Note that eq. (10) constitutes an excellent tool for extracting both the interaction potential and the equation of state directly from measured pair-distribution functions, provided that the range of $V_{\mathrm{sr}}(r)$ is below $2 \sigma$. In that case, we can employ the exact solution to obtain $\beta p$ and $\Omega(\beta p)$ from a linear fit to $\ln \left(\rho^{(1)}(r)\right)$ for $r \lesssim 2 \sigma$, where $V_{\mathrm{sr}}(r)$ vanishes. Then, $V(r)$ follows directly from eq. (10). In higher dimensions the pair-distribution function at small separations cannot be described in such simple terms because even particles in the first correlation shell can exchange positions. Therefore it becomes more involved to extract the pair potential from the knowledge of the pair-distribution function.

As an aside, we note that the constant $\Omega(\beta p)$ obtained from the fit is connected to the chemical potential $\mu$ through $\beta \mu=-\ln (\Omega(\beta p))$. Given that $p$ and $\mu$ can be obtained in such a simple way from $\rho^{(1)}(r)$, the method could be applied for an experimental test of the Gibbs-Duhem relation which, at constant temperature, is equivalent to $\partial p / \partial \mu=\rho_{\mathrm{b}}$. A further interesting implication for experiments arises from the fact that $\rho^{(1)}(r)$ is written as an inverse Fourier transform which allows for a simple calculation of the static structure factor $S(t)=1+\int \exp [-i t r] \rho^{(1)}(r) \mathrm{d} r[2]$. One finds that $S(t)=1+2 \operatorname{Re}\{q(t) /(1-q(t))\}$ for the present system. Note, however, that scattering experiments in $d=1$ are difficult due to the small number of particles involved leading to low scattering intensities.

For our experimental studies we use a dilute aqueous suspension of electrostatically stabilised, polystyrene spheres with a diameter of $\sigma=2.9 \mu \mathrm{m}$ (see fig. 1(a)). The particles are imaged by means of an inverted microscope and particle positions are recorded by video microscopy [8]. The particle motion is confined to a one-dimensional (1D) optical trap which is created with an optical tweezer. For this purpose, a single laser beam is continuously deflected by a pair of galvanometric mirrors with a frequency $f=330 \mathrm{~Hz}$ thus forming a circular line (see fig. 1(b)). The circle is projected with a microscope objective into the sample cell which consists of two $200 \mu \mathrm{m}$ spaced, flat glass substrates. The negatively charged particles are forced towards the likely charged lower glass wall by the vertically incident laser tweezer. 

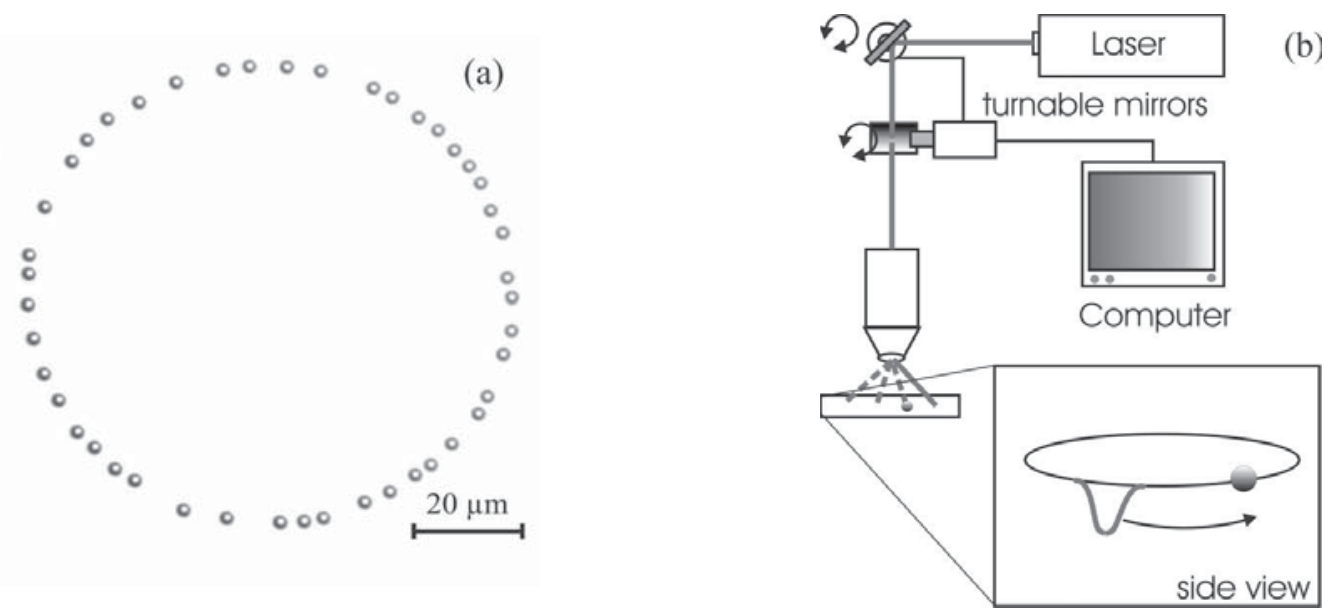

Fig. 1 - (a) Snapshot of $N=45$ electrostatically stabilised, polystyrene spheres with diameter $\sigma=$ $2.9 \mu \mathrm{m}$ in deionised water. The particle motion is limited to a circular line by a ring-shaped optical trap. (b) Experimental setup.

Therefore the equilibrium position of the particles is typically located about $50 \mathrm{~nm}$ above the glass plate. Vertical particle fluctuations are on the order of $5 \%$ of the particle diameter.

Figure 2(a) shows the averaged particle density in the presence of a scanning laser focus. As can be seen, the colloids are effectively confined to a 1D toroidal trap with a rather uniform density distribution along the circumference (fig. 2(a)). It has been demonstrated by Faucheux et al. [9] that at sufficiently high scanning frequencies the particles experience an effective quasi-static potential. Accordingly, at the scanning frequency used in our experiments, i.e. $330 \mathrm{~Hz}$, the particles are in thermal equilibrium and diffuse freely along the laser trap. From the radial particle distribution we obtain the radial trap potential $V_{\text {radial }}(R)$ acting on the colloids (fig. 2(b)). When comparing the width of the potential with the particle diameter, it becomes clear that the spheres cannot pass each other and that indeed our experiment
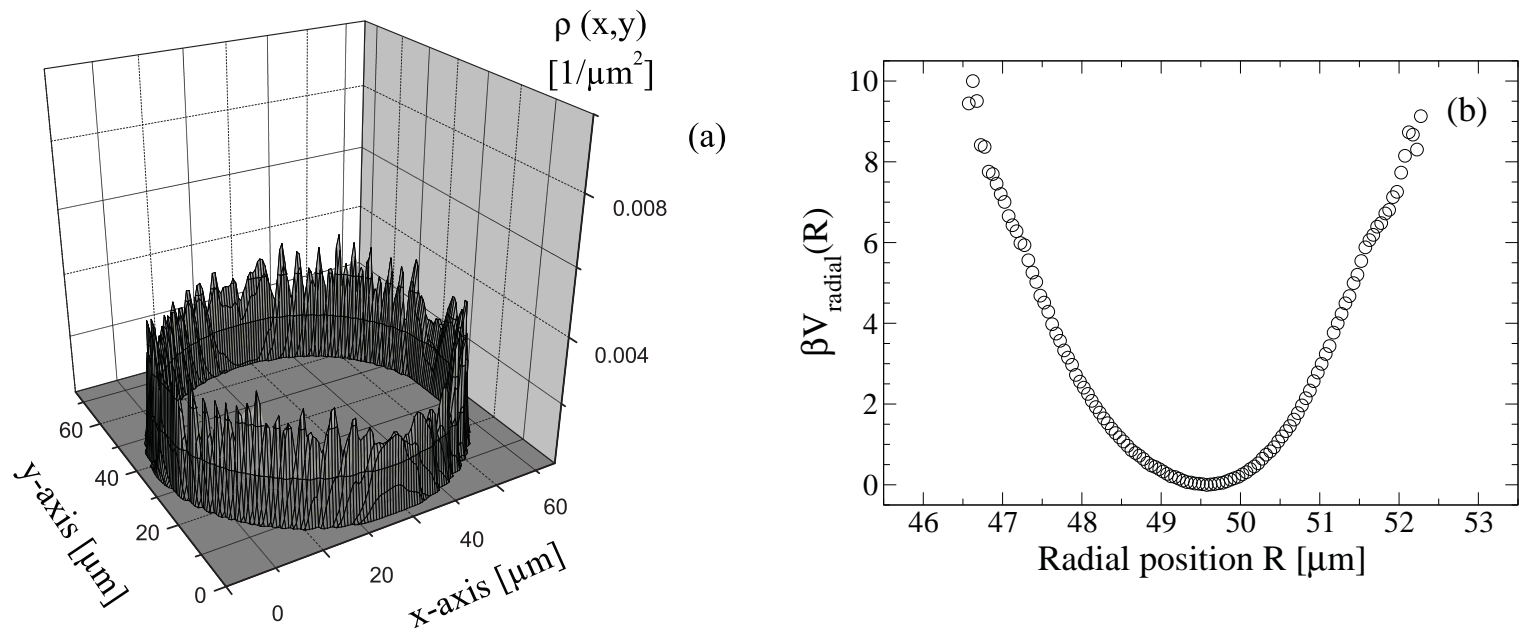

Fig. 2 - (a) Particle density distribution $\rho(x, y)$ for one of our measurements. The particles distribute only, and there equally, along the circular line drawn by the laser focus. (b) An averaged light potential perpendicular to the scanning direction, sampled by the particles due to Brownian motion. 

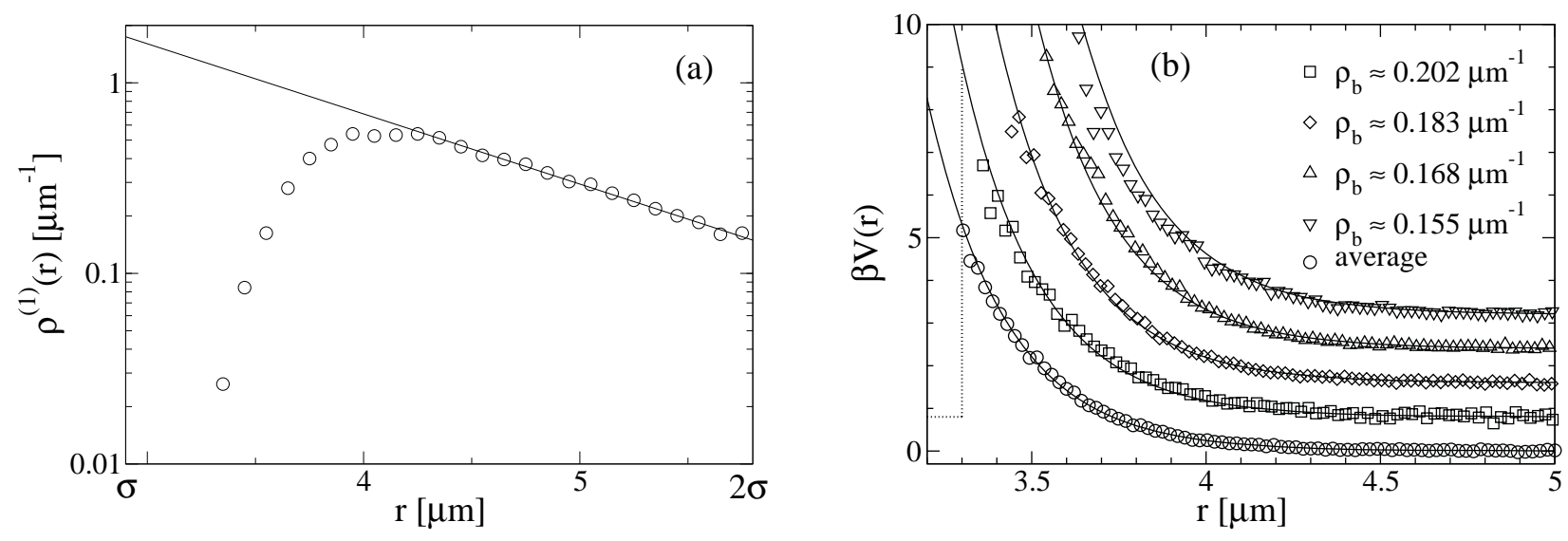

Fig. 3 - (a) Logarithmic plot of $\rho^{(1)}(r)$ for $\rho_{\mathrm{b}} \simeq 0.202 \mu \mathrm{m}^{-1}$. The linear behaviour of $\ln \left(\rho^{(1)}(r)\right)$ confirms that the pair potential $V(r)$ is short ranged. (b) Individual and averaged pair potentials from the measurements. The full lines correspond to the best fit of $V_{\mathrm{DLVO}}(r)$ to the averaged pair potential (circles). The curves have been shifted for clarity along both axes (see dotted line).

is an excellent realisation of a 1D trap. As expected for such systems, the mean-square displacement follows the well-known square-root time dependence which is a unique signature of $1 \mathrm{D}$ systems [10].

In order to vary the line density of particles inside our trap, we performed measurements with $N=45$ colloids trapped on circles with different radii $R \simeq 35.5 \mu \mathrm{m}, 39.0 \mu \mathrm{m}, 42.5 \mu \mathrm{m}$ and $46.0 \mu \mathrm{m}$, respectively. The one-particle density $\rho^{(1)}(r)$ is obtained from the position data of the colloids as a histogram of the distances with the normalisation $1 /(N M \Delta r)$, where $N$ is the number of colloids in the trap, $M$ the number of evaluated snapshots and $\Delta r$ the spatial resolution of the histogram. The distances are measured along the circular confinement of the trap. The influence of the finite curvature is negligible because on the range of the particle interaction (approximately $2 \sigma \simeq 5.8 \mu \mathrm{m}$ ) the linear particle distance differs from that measured along the circle by less than $0.1 \%$, even for the smallest radius of the trap. Concerning finitesize effects, we remark that the correlation length $\xi$ is below $2 \sigma$ even for the highest density as we have checked with the exact solution for $\rho^{(1)}(r)$. Given the length of the circular line it is therefore justified to regard the system with periodic boundary conditions as infinite.

Experimentally, $\rho_{b}$ is determined from the value to which the one-particle density $\rho^{(1)}(r)$ converges towards large distances. From the four measurements we find that $\rho_{\mathrm{b}} \simeq 0.202 \mu \mathrm{m}^{-1}$, $0.183 \mu \mathrm{m}^{-1}, 0.168 \mu \mathrm{m}^{-1}$ and $0.155 \mu \mathrm{m}^{-1}$.

In order to verify the short range of the particle interaction, we confirm that for the different measurements $\ln \left(\rho^{(1)}(r)\right)$ displays linear behaviour at least on the last $\mu \mathrm{m}$ of the interval $[\sigma, 2 \sigma]$. As an example, we show $\ln \left(\rho^{(1)}(r)\right)$ for $\rho_{\mathrm{b}} \simeq 0.202 \mu \mathrm{m}^{-1}$ in fig. 3(a). For each density, this linearisation yields the osmotic pressure $\beta p$ of the colloids and the constant $\Omega(\beta p)$. Equation (10) can then be inverted directly to obtain unambiguously $V(r)$, as shown in fig. 3(b). The potentials from the four measurements agree quite well. Only the results for $\rho_{\mathrm{b}} \simeq 0.155 \mu \mathrm{m}^{-1}$ which was the last measurement shows slight deviations. This is most likely due to the slow increase of airborne ions in the solvent during the measurement.

We aim at finding a single pair potential that describes all measurements. This would enable us to calculate the equation of state and the one-particle density for any given bulk density $\rho_{\mathrm{b}}$. The interaction of charged spherical colloids in a bulk solution (for a review see [11]) has been described by various approaches which are valid in different regimes [12-14]. According to Derjaguin-Landau-Verwey-Overbeek (DLVO) theory [13], the interaction of two 

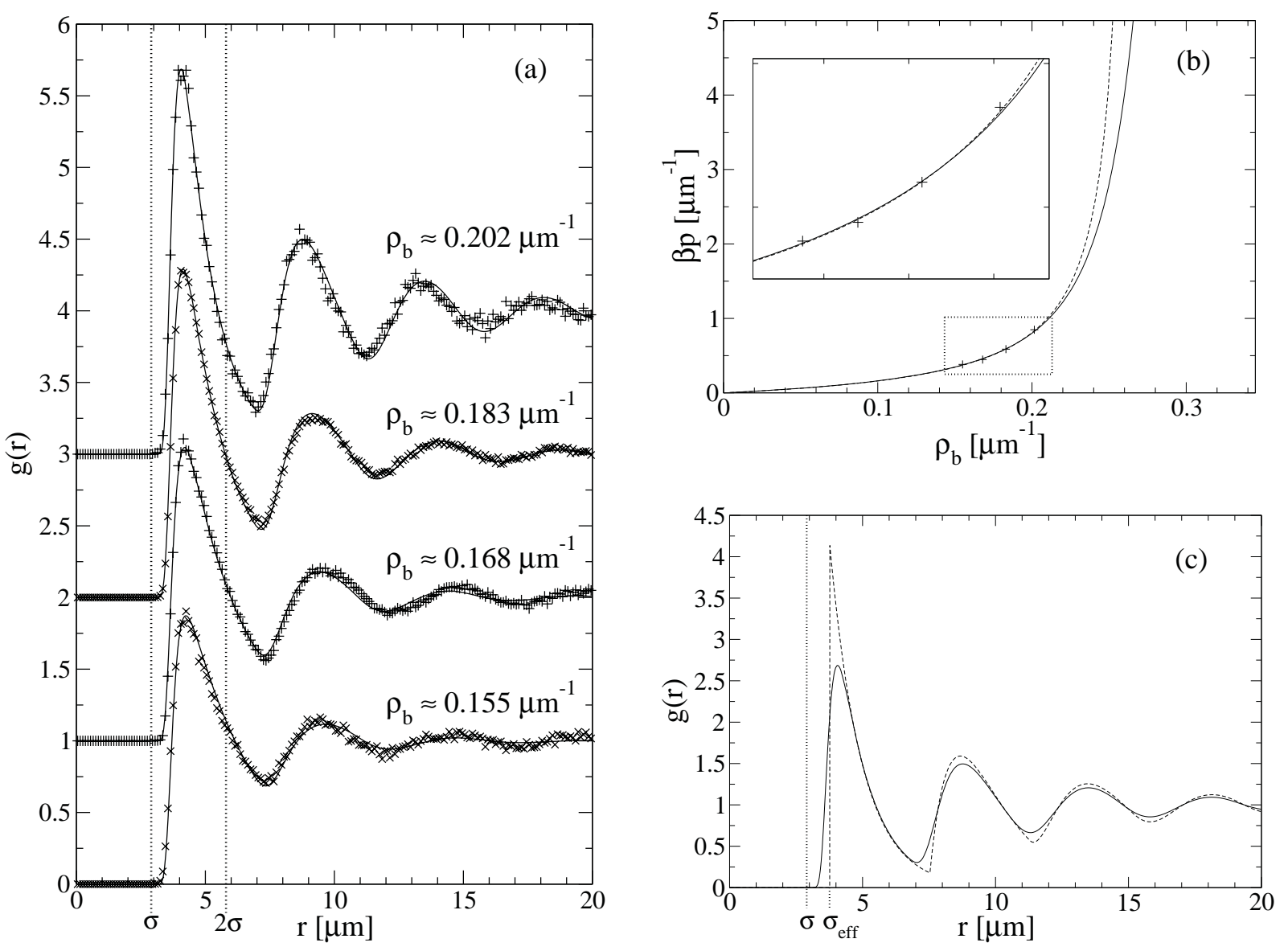

Fig. 4 - (a) Pair-distribution function $g(r)$ from the measurements compared with the analytical calculation (full line). The curves have been shifted for clarity. $\sigma$ is the particle diameter. (b) Pressure of the colloids with interaction $V_{\mathrm{DLVO}}$ (full line). Fit of the equation of state for hard spheres (dashed line). The points represent the pressure from the four measurements obtained through eq. (10). (c) $g(r)$ of the system with interaction $V_{\text {DLVO }}$ (full line) compared to that of a hard-sphere system with effective diameter $\sigma_{\text {eff }}$ (dashed line) at a bulk density $\rho_{\mathrm{b}} \simeq 0.202 \mu \mathrm{m}^{-1}$.

charge-stabilised colloids can be described through a screened Coulomb potential:

$$
\beta V_{\mathrm{DLVO}}(r)=\frac{1}{4} \phi^{2} \frac{\sigma^{2}}{\ell_{\mathrm{B}} r} \exp [-\kappa(r-\sigma)]
$$

where $\ell_{\mathrm{B}} \simeq 7 \AA$ is the Bjerrum length in water at room temperature. The Debye screening length $\kappa^{-1}$ and the normalised surface potential $\phi$ are characteristic for the solvent and the colloidal particles.

The best fit of $V_{\mathrm{DLVO}}(r)$ to the averaged pair potential from the four measurements is obtained for $\kappa^{-1} \simeq 244 \mathrm{~nm}$ and $\phi \simeq 0.174$, and is in good agreement with the experimental data (see full lines and circles in fig. 3(b)). The effective charge of the colloids can be obtained as $Z^{\text {eff }}=\ell_{\mathrm{B}}^{-1}(\sigma / 2)(1+\kappa \sigma / 2) \phi[15]$. For our data, we obtain $Z^{\text {eff }} \simeq 2500$ which is in good agreement with the result of an independent measurement $\left(Z^{\text {eff }} \simeq 2250\right)$ where the pair potential was obtained from $g(r)$ by inversion of the Ornstein-Zernike equation in $d=2[16]$.

Using the fitted pair potential $V_{\mathrm{DLVO}}(r)$, we can now calculate the equation of state and the one-particle density $\rho^{(1)}(r)$ for arbitrary values of $\rho_{\mathrm{b}}$ as described previously. The results for the pair-distribution function $g(r)=\rho^{(1)}(r) / \rho_{\mathrm{b}}$ are shown in fig. 4(a). The analytical curves 
are in very good agreement with the experimental data. We have marked the interval $[\sigma, 2 \sigma]$ to emphasise that only experimental data from this interval have been used for determining the common pair potential and hence the analytical curves for the whole function $g(r)$.

The result for the equation of state is shown in fig. 4(b). As a consistency check, we show the results for $\beta p$ as obtained from the four measurements through eq. (10). Finally, we compare results for a system with DLVO interaction to that of a hard-sphere system, which corresponds to $V(r) \equiv 0$ in eq. (5). The equation of state follows from eq. (1): $\beta p=$ $\rho_{\mathrm{b}} /\left(1-\sigma \rho_{\mathrm{b}}\right)$. If we consider $\sigma$ of the hard-sphere system as a free parameter, this equation can be fitted to the equation of state previously obtained, yielding an effective diameter $\sigma_{\text {eff }} \simeq 3.76 \mu \mathrm{m}$. From the bulk properties, the investigated colloidal system can hardly be distinguished from the effective hard-sphere system in a wide range of $\rho_{\mathrm{b}}$ (see fig. 4(b)). When local properties are required, it turns out that the description through a hard-sphere system leads to results that differ significantly from the experimental findings, as can be seen from fig. 4(c). The mapping onto an effective hard-sphere system has been carried out previously for charge-stabilised colloids in two dimensions [17] with qualitatively similar agreement.

In conclusion, we have introduced eq. (10), which is part of the exact statistical mechanics solution of the one-dimensional system where only next neighbours interact, as an excellent tool for extracting the pair potential $V(r)$ and the pressure $\beta p$ (hence the equation of state) from experimentally measured pair-distribution functions $g(r)$, provided $V(r)$ is short ranged. We have applied the method to colloids confined to a one-dimensional laser trap and find good agreement between the experiment and the exact solution assuming the pair interactions to be of the DLVO type.

HHG and RR thank K. MECKE for stimulating discussions and helpful comments.

\section{REFERENCES}

[1] Brenan G. P. and Evans R., Mol. Phys., 73 (1991) 789.

[2] Hansen J.-P. and McDonald I. R., Theory of Simple Liquids (Academic Press, London) 1986.

[3] Lyubartsev A. P. and Laaksonen A., Phys. Rev. E, 52 (1995) 3730.

[4] Lobaskin V., Brunner M., Bechinger C. and von Grünberg H. H., J. Phys. Condens. Matter, 15 (2003) 6693.

[5] Gürsey F., Proc. Cambridge Philos. Soc., 46 (1950) 182.

[6] Salsburg Z. W., Zwanzig R. W. and Kirkwood J. G., J. Chem. Phys., 21 (1953) 1098.

[7] Võ T. T. M., Chen L.-J. and Robert M., J. Chem. Phys., 119 (2003) 5607.

[8] Baumgartl J. and Bechinger C., Europhys. Lett., 71 (2005) 487.

[9] Faucheux L. P., Stolovitzky G. and Libchaber A., Phys. Rev. E, 51 (1995) 5239.

[10] Lutz C., Kollman M. and Bechinger C., Phys. Rev. Lett, 93 (2004) 026001.

[11] Belloni L., J. Phys. Condens. Matter, 12 (2000) R549.

[12] Derjaguin B., Kolloid-Z., 69 (1934) 155; Acta Physicochim., 10 (1939) 333.

[13] Verwey E. J. W. and Overbeek J. Th. G., Theory of the Stability of Lyophobic Colloids (Elsevier, Amsterdam) 1948.

[14] Bell G. M., Levine S. and McCartney L. N., J. Colloid Interface Sci., 33 (1970) 335.

[15] Belloni L., Colloids Surf. A, 140 (1998) 227.

[16] Brunner M., Bechinger C., Strepp W. and von Grünberg H. H., Europhys. Lett., 58 (2002) 926.

[17] Brunner M., Bechinger C., Herz U. and von Grünberg H. H., Europhys. Lett., 63 (2003) 791. 